\title{
BK Knockout by TALEN-Mediated Gene Targeting in Osteoblasts: KCNMA1 Determines the Proliferation and Differentiation of Osteoblasts
}

\author{
Hongya Heil, ${ }^{1,5}$ Jianjun Gao ${ }^{2,5}$, Jibin Dong', Jie Tao ${ }^{3}$, Lulu Tian', Wanma Pan', Hongyu Wang ${ }^{4}$, and \\ Xuemei Zhang ${ }^{\text {1,* }}$
}

\begin{abstract}
Large conductance calcium-activated potassium (BK) channels participate in many important physiological functions in excitable tissues such as neurons, cardiac and smooth muscles, whereas the knowledge of BK channels in bone tissues and osteoblasts remains elusive. To investigate the role of BK channels in osteoblasts, we used transcription activator-like effector nuclease (TALEN) to establish a BK knockout cell line on rat ROS17/2.8 osteoblast, and detected the proliferation and mineralization of the BK-knockout cells. Our study found that the BKknockout cells significantly decreased the ability of proliferation and mineralization as osteoblasts, compared to the wild type cells. The overall expression of osteoblast differentiation marker genes in the BK-knockout cells was significantly lower than that in wild type osteoblast cells. The BK-knockout osteoblast cell line in our study displays a phenotype decrease in osteoblast function which can mimic the pathological state of osteoblast and thus provide a working cell line as a tool for study of osteoblast function and bone related diseases.
\end{abstract}

\section{INTRODUCTION}

Large conductance calcium-activated potassium (BK) channels, also known as MaxiK/KCa1.1 channels, are encoded by KCNMA1 gene and widely expressed in most cells and tissues (Bentzen et al., 2014; Contreras et al., 2013). The uniqueness of BK channels is that it can be regulated by both membrane voltage and local cytosolic calcium. BK channel in excitatory

\footnotetext{
1Department of Pharmacology, School of Pharmacy, Fudan University, Shanghai 201203, China, ${ }^{2}$ Department of Bone Metabolism, Institute of Radiation Medicine, Fudan University, Shanghai, 200032, China, ${ }^{3}$ Department of Nephrology, Putuo Hospital, Shanghai University of Traditional Chinese Medicine, Shanghai 200062, China, ${ }^{4}$ Department of Pharmacy, Shenyang Pharmaceutical University, Shenyang 110016, China, ${ }^{5}$ These authors contributed equally to this work.

*Correspondence: xuemzhang @fudan.edu.cn
}

Received 5 February, 2016; revised 21 May, 2016; accepted 23 May, 2016; published online 21 June, 2016

Keywords: BK channels, knockout, osteoblast, TALEN tissues has been widely studied. In smooth muscle cells, BK channels regulate vascular contractility in coordination with ryanodine receptor and L-type $\mathrm{Ca}^{2+}$ channels by increasing intracellular calcium concentration (Sandow and Grayson, 2009; Wu and Marx, 2010). In hair cells, BK channels can be activated by the intracellular calcium increase induced by Ltype voltage-dependent $\mathrm{Ca}^{2+}$ channels, and promote membrane oscillation (Issa and Hudspeth, 1994). In nervous system, BK channel neuron is related with action potential firing and transmitter release (Jin et al., 2000). Due to few studies, our understanding of BK channel in non-excitatory tissue such as bone cells, tumor cells and immune cells is very limited (Morera et al., 2015). In human, BK channels can be detected in (Hirukawa et al., 2008) in osteosarcoma cells (MG-63, SaOS-2, and G292 cells) and osteogenic precursor cells (C1 cells). In a study of human osteoblast-like cells, Henney et al. (2009) found tetraethylammonium (TEA), a non-specific potassium inhibitor, had a biphasic effect on cell growth with mitogenic property at low concentration and inhibitory at high concentration, indicating the presence of BK channels. BK knockout mice appeared to be osteopenic, with reduced bone mineral density in long bones and elevated porosity of trabecular meshwork (Sausbier et al., 2011), indicating a role of BK channels in bone development. However, the role of BK channel in osteoblasts is far from clear. Suppressing BK channel expression by knocking down KCNMA1 gene with shRNA inhibited cell proliferation and osteogenic differentiation in human bone marrow-derived mesenchymal stem cells (Zhang et al., 2014). However, RNA interference-mediated knockdown could not completely abolish target gene expression and the leaky expression still could contribute BK channel function in the study. Gene knockout is the ideal way to completely eliminate gene expression and analyze gene function.

The advent of gene modification techniques such as Zinc finger nuclease (ZFN), transcription activator-like effector nuclease (TALEN) and clustered regularly interspaced short palindromic repeats (CRISPR)-Casenzymes in recent years has greatly prompted the wide application of genomic target modification in both eukaryotic cells and experimental animals. These techniques make it possible to manipulate gene expression at genomic level, especially for our studying BK channels function in osteoblasts. Considering the possible role of osteoblasts in bone formation and bone related diseases (Long, 2012), we intended to investigate the function of BK channels in osteo- 
blasts by eliminating BK channels in osteoblasts completely. Using TALEN gene targeting technique describes in publications (Chen et al., 2014; Tokuda and Furuse, 2015),we performed KCNMA1 gene (NM_031828) knockout on rat ROS17/ 2.8 osteoblasts, and successfully established a BK knockout cell line whose phenotype is decreased ability of proliferation and mineralization compared to the original wild type osteoblasts. BK channel could be a therapeutic target for the treatment of bone-related diseases and the BK-knockout cell line we established can be a potent tool for study of osteoblast function.

\section{MATERIALS AND METHODS}

\section{Cell culture and reagents}

ROS17/2.8 cells, a rat osteoblastic cell line, were a kind gift from Dr. Sandra Guggino (Johns Hopkins University, USA), and MC3T3-E1 cells, a mouse osteoblastic cell line, from Dr Sun Xun (Fudan University, China). ROS17/2.8 cells were cultured in Ham's F-12 nutrient mixture medium (Sigma-Adrich), MC3T3-E1 cells were cultured in alpha-MEM medium. Both medium contained $\mathrm{NaHCO}_{3}$ and supplemented with $10 \%$ fetal bovine serum (FBS, Gibco). Cells were cultured in an atmosphere of $5 \%$ carbon dioxide and $95 \%$ air at $37^{\circ} \mathrm{C}$, and subcultured about every 2 days. For experiments, the cells were trypsinized at about $90 \%$ confluence and seeded in 6 -well plates at a density of $2.5 \times 10^{4} / \mathrm{ml}$.

Rat anti-BK polyclonal antibody (AP107) were purchased from Alomone (Israel). E.Z.N.A. ${ }^{\circledR}$ Plasmid Mini Kit were purchased from Yeasen Biology (China). Genomic DNA Kit was purchased from Tiangen Biology (China).

\section{TALENs design and construction}

A Fast TALE ${ }^{\text {TM }}$ Assembly kit from SiDanSai biotechnology (China) was used to construct TALENs according to the detailed protocol provided by the manufacturer (Chen et al., 2014; Wu et al., 2014). Briefly, the left arm and right arm of TALENs that target KCNMA1 gene were designed on the website (https://talent.cac.cornell.edu/). With the TAL-Multimers and vectors chosen from the kit provided by the manufacturer, polymerase chain reaction (PCR)-amplify were performed to assemble TALENs. After PCR products were transformed into $\mathrm{DH} 5 \alpha, 12$ clones for each arm were picked for TALEN plasmid extraction using The E.Z.N.A. ${ }^{\circledR}$ Plasmid Mini Kit. Purified plasmids were digested with Pstl and BamHI enzyme and sequenced by SanGonBiotech (China).

PCR amplification and sequence analysis of genomic DNA Genomic DNA were extracted from the colonies using a Genomic DNA kit from TIANGEN (China), and subjected to PCR to amplify TALEN targeting on KCNMA1. The forward primer is 5'-ATATCCACGCGAACCATCTCAGCCT- ${ }^{\prime}$ and the reverse primer is $5^{\prime}$-GGCCGTAGCCACCAATAACCCTACA-3'. The PCR amplification was run on CFX96 Real-Time System thermal cycler (Bio-Rad Laboratories, USA) with initial activation for $30 \mathrm{~s}$ at $95^{\circ} \mathrm{C}$ and followed by 42 cycles of denaturation $\left(95^{\circ} \mathrm{C}, 5\right.$ $\mathrm{s})$, annealing $\left(60^{\circ} \mathrm{C}, 34 \mathrm{~s}\right)$, and extension $\left(72^{\circ} \mathrm{C}, 30 \mathrm{~s}\right)$. The PCR products were analyzed by SanGonBiotech (China).

\section{Establishment of KCNMA1 knockout clones}

To establish BK knockout clones, a pair of TALEN plasmids was transfected into ROS 17/28 cells and MC3T3-E1 cells in 6 well plates using Lipofectamine 2000. The $4 \mu \mathrm{g} / \mathrm{ml}$ Puromycin was added for selection on the second day of transfection.
Table 1. Primers for real time RT PCR

\begin{tabular}{ll}
\hline Gene & Primer \\
\hline Runx2 & Forward: 5'-CCCCATCCATCCATTCCACC-3' \\
& Reverse: 5'-GGCAGTGTCATCATCTGAAATACGC-3' \\
OCN & Forward: 5'-ATGTCCAAGCAGGAGGGCAGTA-3' \\
& Reverse: 5'-CCAAGTCCATTGTTGAGGTAGCG-3' \\
OPN & Forward: 5'-AGGAGTTTCCCTGTTTCTGATG-3' \\
& Reverse: 5'-TGGTCTTCCCGTTGCTGTC-3' \\
GAPDH & Forward: 5'-ATGACCACAGTCCATGCCATC-3' \\
& Reverse: 5'-CCTGCTTCACCACCTTCTTG-3' \\
\hline
\end{tabular}

Growing clones were re-plated at low density and allowed them to recover and grew for 1 week, resulting in the formation of distinct single colonies (Lu et al., 2013). Colonies were picked for screening KCNMA1 knockout by immunofluorescent staining and DNA sequencing individual clones.

\section{Quantitative PCR for gene expression}

mRNA expression were analyzed by real-time RT PCR as described elsewhere (Chen et al., 2015). Total RNA of BK knockout clones and wild type cells were extracted by using RNAiso Plus reagent (TaKaRa, China) and First-strand cDNA were synthesized using the PrimeScript RT Master Mix (TaKaRa, China). A total of $2 \mu \mathrm{l}$ of the first-strand cDNA solution was used in combination with the SYBR ${ }^{\circledR}$ Premix Ex Taq $^{T M}$ solution (TaKaRa, China) for real-time (RT)-PCR to quantify specific gene expression. The RT-PCR was run on CFX96 Real-Time System thermal cycler. The primers used for RT-PCRs are listed in Table 1. Each reaction was repeated three times. The gene expression levels were analyzed by $\Delta \Delta C_{t}$ method and normalized to GAPDH. Data were expressed as fold change to control.

\section{Immunofluorescent staining}

BK knockout clones and wild type cells were seeded on cell slides. At $70 \%$ confluent, cells were fixed in $4 \%$ paraformaldehyde for $15 \mathrm{~min}$. Following washes with PBS, cells were permeabilized in $0.1 \%$ Triton $\mathrm{X}-100$ for $10 \mathrm{~min}$ and blocked in $3 \%$ bovine serum albumin (BSA) in PBS buffer for $1 \mathrm{~h}$ at room temperature. The primary antibody for BK protein (1:200, Abcam, UK) was incubated overnight at $4^{\circ} \mathrm{C}$. The secondary AlexaFluor647-conjugated anti-mouse antibody (Jackson ImmunoResearch, USA) was used at a 1:200 dilution for 1 hour at room temperature. Images were captured using a Leica TCS SP5 confocal microscopy (Leica, Germany)

\section{Osteoblast mineralization}

ROS17/2.8 cells were seeded into 48-well plate. When they were almost confluent, beta- glycerophosphate $(10 \mathrm{mM})$ and Iascorbicacid 2-phosphate $(50 \mu \mathrm{g} / \mathrm{ml})$ were added into the growth medium to induce mineralization. Cells grown in regular medium were considered as a control. The medium were changed every three days. On day 10 , cells were fixed with $4 \%$ paraformaldehyde for 10 min after washing with PBS and used for Alizarin Red S staining.

\section{Alizarin Red S staining and quantification}

Cells were gently washed with PBS three times and then $500 \mu \mathrm{l}$ of $0.1 \%$ Alizarin Red S (Yeasen, China) was added to dishes to stain cells for $15 \mathrm{~min}$ at room temperature. After aspiration 
Construct a BK Knockout Cell Line by TALEN Techonology

Hongya Hei et al.

Table 2. The sequences and linked vectors of five TALENs

\begin{tabular}{lll}
\hline Name & Sequence & Vector \\
\hline L1 & CTTCATCATCTTGCTCTG & CMV-SP6-3xFlag-NLS-N'-[TALE]-1/2G-C'-Fokl-IRES-puro-pA \\
L2 & CTTCATCATCTTGCTCTGG & CMV-SP6-3xFlag-NLS-N'-[TALE]-1/2G-C'-Fokl-IRES-puro-pA \\
R1 & GGCAGCAAACGGTCCACA & CMV-SP6-3xFlag-NLS-N'-[TALE]-1/2A-C'-Fokl-pA \\
R2 & GGCAGCAAACGGTCCAC & CMV-SP6-3xFlag-NLS-N'-[TALE]-1/2C-C'-Fokl--pA \\
R3 & GGCAGCAAACGGTCCACAG & CMV-SP6-3xFlag-NLS-N'-[TALE]-1/2G-C'-Fokl--pA \\
\hline
\end{tabular}

of the unincorporated dye, the cells were washed three times with distilled water with shaking for $5 \mathrm{~min}$ each time (Yu et al., 2013). Matrix mineralization was quantified by extracting the alizarin Red S staining with 100 mM cetylpyridinium chloride solution. The absorbance was measured at $572 \mathrm{~nm}$.

\section{CCK8 cell proliferation assay}

BK knockout cells and wild type osteoblasts were plated in 96well plates at a density of 2000 cells per well. On day 2, 3, 4, 5, 7 and 9 after seeding, the cell proliferation assay was performed by the addition of $10 \mu$ l CCK8 solution (Yeasen, China) to each well, followed by an incubation at $37^{\circ} \mathrm{C}$ for $2 \mathrm{~h}$. Absorbance was read out at a wavelength of $450 \mathrm{~nm}$ using a microplate reader (TECAN infinite m200 pro, Switzerland).

\section{Statistical analysis}

Experimental results are expressed as mean \pm standard error of the mean (S.E.M). Statistical significance was assessed by using a Student's paired t test when there were only two groups involved and by one-way ANOVA with Bonferroni post-hoc tests for experiments with three or more groups. $\mathrm{P}<0.05$ was considered statistically significant. All data analyses were performed using the software GraphPad PRISM 5 (GraphPad Software, Inc., USA).

\section{RESULTS}

\section{Construction of TALENs targeting KCNMA1 gene}

To construct TALENs targeting KCNMA1 in osteoblasts, we designed 2 left arms and 3 right arms TALENs (Fig. 1A). The sequences and vectors used were listed in Table 2 . TALENs constructs from PCR amplification were transformed into $\mathrm{DH} 5 \alpha$ which grow in LB agar plate. Four clones for each construct were picked from LB plates for plasmid extraction. Plasmids were digested with enzymes Pstl and BamHI. The theoretic digestion fragments should be $4.8 \mathrm{~kb}+2.2 \mathrm{~kb}$ for $L 1,4.8 \mathrm{~kb}+$ $2.3 \mathrm{~kb}$ for $L 2,3.6 \mathrm{~kb}+2.2 \mathrm{~kb}$ for $\mathrm{R} 1,3.6 \mathrm{~kb}+2.2 \mathrm{~kb}$ for $\mathrm{R} 2$ and $3.6 \mathrm{~kb}+2.3 \mathrm{~kb}$ for R3 (Figs. 1B and 1C). Only TALENs plasmids which showed the expected fragment size were selected for further experiments.

The 5 TALEN plasmids selected were combined into 6 pairs following as L1/R1, L1/R2, L1/R3, L2/R1, L2/R2 and L2/R3 and used for osteoblast transfection. Upon ROS17/2.8 transfection and puromycin treatment, only the cells with L1/R2 pair survived and grew into distinct colonies. The genomic DNA of each colony were extracted for TA cloning (Yen et al., 2015) to confirm the targeting efficiency. We found 4 were mutated out of the 27 colonies chosen for TA cloning, reaching an efficiency of $29.6 \%$. Thus, we successfully constructed a pair of TALENs that target KCNMA1 gene with a targeting efficiency of $29.6 \%$ in ROS17/2.8.
A
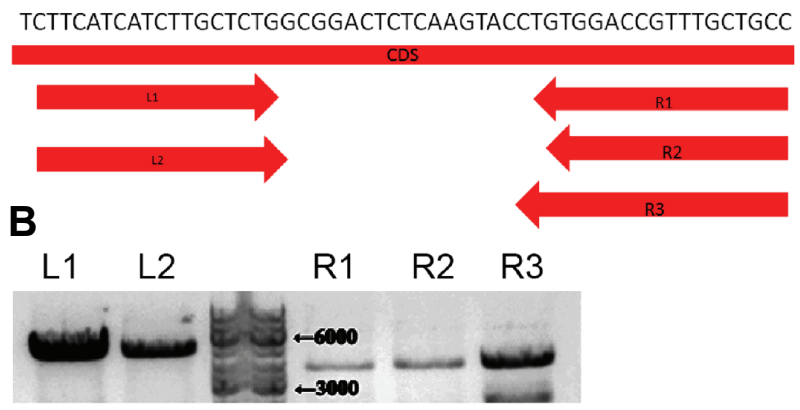

C
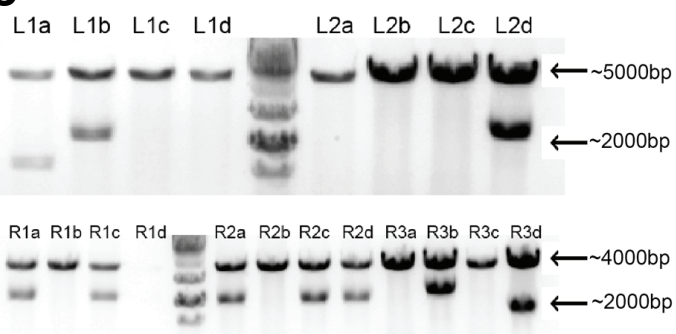

Fig. 1. Construction of TALENs targeting KCNMA1 gene. (A) Fast TALE $^{\text {TM }}$ kit was used to construct gene knockout. The design of TALEN binding site on KCNMA1 gene was shown, with the left arms being $L 1$ and $L 2$, and the right arms being $R 1, R 2$ and $R 3$. (B) Agarose gel DNA electrophoresis showed the five TALEN plasmids constructed. (C) With enzyme digestion, L1b (4.8 kb + 2.2 kb), L2d (4.8 $\mathrm{kb}+2.3 \mathrm{~kb}), \mathrm{R} 1 \mathrm{a}(3.6 \mathrm{~kb}+2.2 \mathrm{~kb}), \mathrm{R} 2 \mathrm{a}(3.6 \mathrm{~kb}+2.2 \mathrm{~kb})$ and R3b $(3.6 \mathrm{~kb}+2.3 \mathrm{~kb})$ were identified as the targeting TALENs plasmids.

\section{Establishment of BK-knockout clones in osteoblasts} Using the L1/R2 pair of TALENs, we transfected ROS17/2.8 osteoblasts, aiming to create BK knockout cells. Upon cell colonies growing up in the culture dish under puromycin selection, they were typsined and diluted into single cell suspension, and seeded into 96-well plates for monoclonal cell culture. All the growing clones were screened for KCNMA1 knockout by DNA sequencing as shown in Fig. $2 \mathrm{~A}$ in which overlapping peaks lie between and after the targeting site, suggesting a successful TALENs targeting. KCNMA1 knockout clones were amplified further to establish a cell line which would be subjected to cell phenotyping. The abolishment of BK expression in BKknockout clones was confirmed by Western blot at protein level (Fig. 2B) and no KCNMA1 transcription detected by RT-PCR (Fig. 2C). We also performed Immunofluorescence also showed the complete loss of BK protein in the BK knockout clones (Fig. 
A

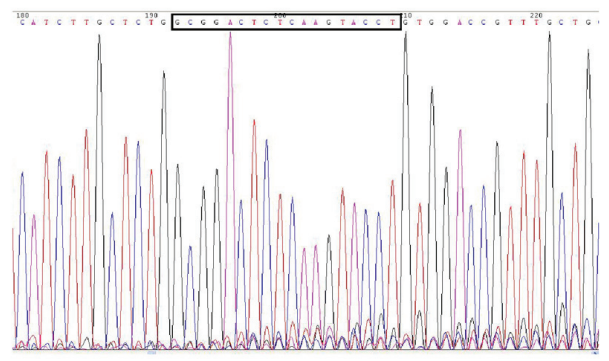

B

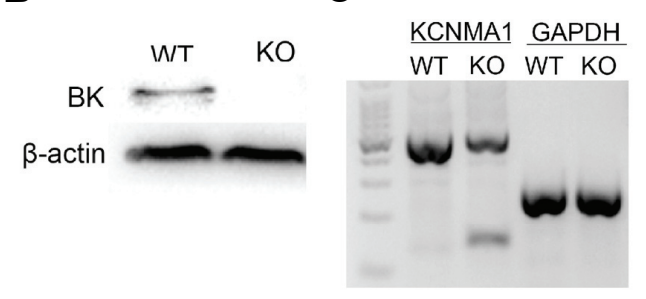

D
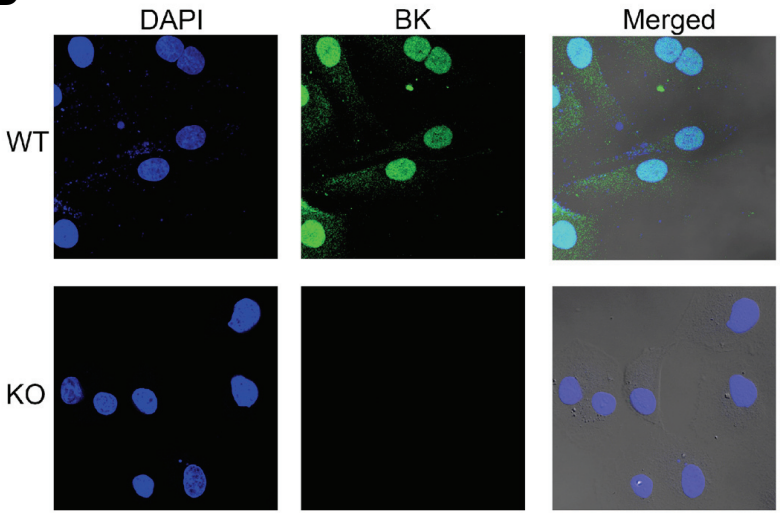

Fig. 2. Establishment of BK-knockout clones in ROS17/2.8 cells. (A) DNA Sequencing map for a BK knockout clone. Overlapping peaks located between and after the targeting site. (B) Western blot analysis showed the expression of BK protein in wild type (WT) and KCNMA1 knockout clones. (C) PCR to measure BK mRNA level. The theoretical size of PCR product for KCNMA1 gene is near 400 bp, KO clones' product was near 500 bp. (D) Immunofluorescence staining of BK channels in wild type (WT) and KCNMA1 knockout (KO) osteoblastic clones. Scale bar $=5 \mu \mathrm{m}$.

2D). We also performed the TALEN on MC3T3-E1 osteoblasts and successfully depleted BK expression (Supplementary Fig. S1).

\section{BK knockout inhibited osteoblast proliferation}

Under microscope, we found the morphology of ROS17/2.8 cells changed from spindle shape into ellipse, and MC3T3-E1 cells having longer feet and smaller body with BK knockout (Fig. $3 \mathrm{~A}$ ), indicating a phenotype change after BK channel depletion on osteoblasts.

To characterize the BK knockout osteoblast clones, we performed CCK-8 proliferation assay to test the effect of BK knockout on cell proliferation. We found that both ROS17/2.8 cells and MC3T3-E1 cells were inhibited in their ability of prolif-
A
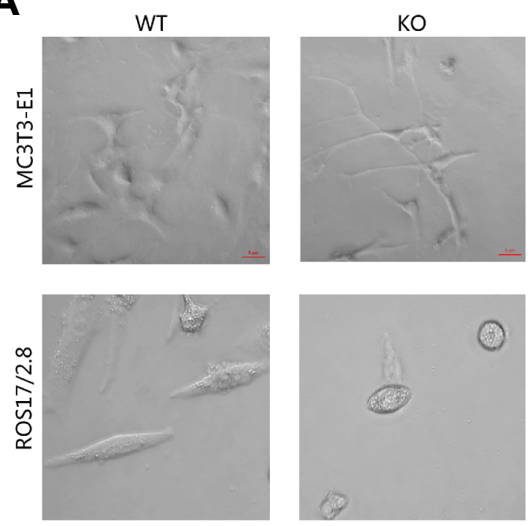

B
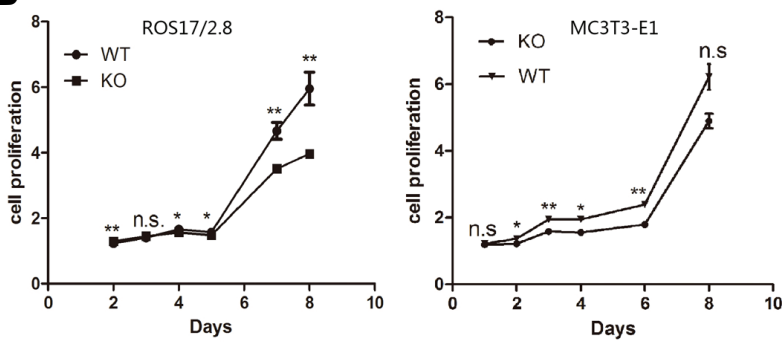

Fig. 3. BK knockout inhibited osteoblast proliferation. (A) The morphology of ROS17/2.8 cells and MC3T3-E1 cells after knocking out BK channels. (B) The proliferation of KCNMA1-knockout cells and wild type cells were assayed by CCK- 8 kit. Cells were collected every $24 \mathrm{~h}$, and the absorbance was measured at $450 \mathrm{~nm}$. The left panel was for ROS17/2.8 cells proliferation, the right panel showed MC3T3-E1 cells' proliferation.

eration (Fig. 3A) with BK knockout, compared to the wild type osteoblasts

\section{BK knockout prevented osteoblast differentiation}

To further characterize BK knockout osteoblast cell line, we studied the cell ability of differentiation towards osteocytes upon induction. Alizarin Red S staining showed that the mineralized nodule formation in BK knockout osteoblasts was significantly suppressed compared to that in the wild type cells (Fig. 4A), indicating that the mineralization ability of BK-knockout ROS17/ 2.8 cell line was decreased significantly with gene depletion. We also examined the changes of genes involved in osteoblast differentiation. These genes we tested by RT-PCR in the current study are Runt-related transcription factor (Runx2) - an osteogenic transcription factor, and two osteoblast markers osteocalcin (OCN) and osteopontin (OPN). Quantified by real time-PCR, the mRNA expression of Runx2, OCN and OPN significantly decreased in ROS17/2.8 BK knockout osteoblasts compared to those in the wild type cells, indicating an impaired differentiation ability of BK knockout osteoblasts.

\section{DISCUSSION}

In the current study, we designed and constructed a pair of TALENs that target KCNMA1 gene and established a BK knockout ROS17/2.8 osteoblast cell line. The BK-knockout 
A

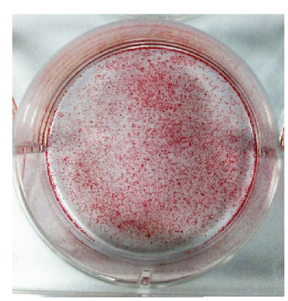

B

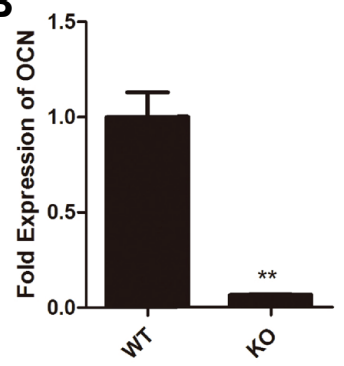

KO
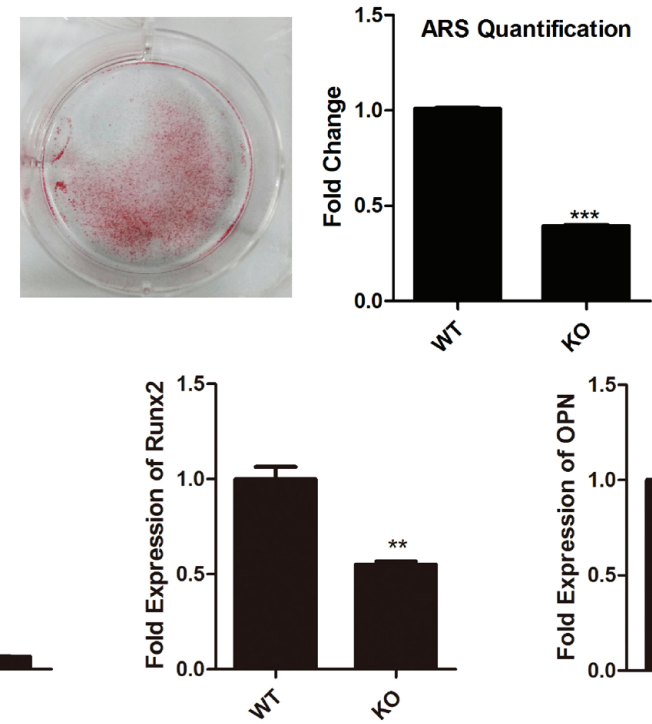

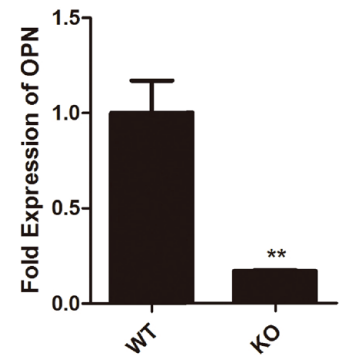

Fig. 4. BK knockout prevented osteoblast differentiation. (A) Formation of mineralized nodules in KCNMA1 knockout cells and wild type cells. Cells were stained with Alizarin Red $S$ after cultured in mineralizationinduction medium for 10 days. Alizarin Red S were extracted by cetylpyridinium chloride and quantified by measuring absorbance at $562 \mathrm{~nm}$. ( $\mathrm{n}$ $\left.=3,{ }^{* \star *} P<0.001\right)(\mathrm{B}) \mathrm{mRNA}$ expression of osteogenic transcription factors (Runx2) and osteoblast markers (osteocalcin (OCN), and osteopontin (OPN) ) were quantified by real-time RT-PCR. mRNA levels of differentiation markers in KCNMA1 knockout cells were much lower than that in the wild type cells $\left(n=3,{ }^{\star \star} P<0.01\right)$. osteoblasts displayed low proliferation and impaired mineralization ability. Data from our study provide further evidence that BK channel is critical for osteoblast function.

BK channels can be found in smooth muscle cells, hair cells, neuron cells and cardiomyocytes and execute diverse physiological functions. BK channels regulate smooth muscle tone by changing intracellular calcium sensitivity (Petkov, 2014). In neuron cells, BK channels participate in cell excitability through regulation of neurotransmitter release (Mehranfard et al., 2015). In these excitable cells, BK channels affect cell signal transduction and viability mainly through the regulation of membrane action potentials. Recently, noncanonical roles of ion channels in diverse non-excitable cell types, including astrocytes, NG2 cells, microglia, macrophages, and cancer cells are reviewed by Black and Waxman (2013), In non-excitable cells such as tumor cells, bone cells and kidney epithelium, it is reported that $\mathrm{BK}$ channels regulate $\mathrm{K}^{+}$homeostasis and cell volume (Morera et al., 2015). Henney et al. (2009) found TEA had a biphasic effect on human osteoblast-like cell growth, which might attribute to the fact that TEA is a non-specific potassium inhibitor. Knocking out KCNMA 1 by TALEN in the current study completely eliminates the influence of channels inhibitors per se. and provides a stable cell line to study BK channel function in osteoblasts.

The BK knockout osteoblasts we established demonstrate a phenotype different from the original wild type cells. The morphology of both ROS17/2.8 and MC3T3-E1 changed greatly compared with wild type. Cell morphology is closely related to cytoskeleton structure and cell function. It was reported that BK channels have physical and functional interactions with actin cytoskeleton in hippocampal pyramidal neurons, VSMCs and podocytes (Huang et al., 2002; Kim et al., 2010; Yamamura et al., 2012). Cell morphology change may also subsequently affect cell function. In our study, the changes in morphology lead to decreased differentiation ability. In addition to the morphological changes, BK knockout osteoblasts grow slow and the differentiation disability impaired, but the underlying mechanism could not be answered in the current study. Several studies on BK channel in cell proliferation on other types of cells could give us some clues. In vascular smooth muscle cells, inhibiting BK channels by tungstate reduced cell proliferation that involved the activation of ERK $1 / 2$ phosphorylation (Fernandez-Marino et al., 2015). Silencing BK channels in bone mesenchymal stem cells (BMSC) inhibited cell proliferation via activating cyclin D1, cyclin E, and p-ERK1/2 (Zhang et al., 2014). Besides, the cyclin D1 and CDK4 complex can phosphorylate Runx2 and target it for ubiquitin-mediated proteasomal degradation (Shen et al., 2006). The significant decrease of Runx2 in BK knockout cells from our study suggests a role of BK channels in cell cycle regulation in osteoblasts. The cell cycle regulation is field of interest for our continuing study of BK channels. The down-regulation of OCN and OPN osteoblastic makers paralleled with the change in Runx2 expression, which might come from the fact that Runx2 can trigger the expression of OCN and OPN (Bruderer et al., 2014).

The change in phenotypes after BK channel knockout in osteoblasts suggests a role of BK channels in bone remodeling, a process characterized by the balance between osteoblasts and osteoclasts. Although animal models of osteoporosis such as ovariectomy have been widely used to study pathological mechanism of this bone remodeling disease, cell models have not been well established. The BK-knockout osteoblastic cell line we established in the current study demonstrates a phenotype of low proliferation and mineralization abilities and might mimic the pathological condition of osteoblasts, thus provides a working tool to study bone remodeling. In conclusion, TALEN genomic editing in cells helped us to establish a BK channel knockout osteoblast cell line which facilitates the function study of disease-related genes.

Note: Supplementary information is available on the Molecules and Cells website (www.molcells.org).

\section{ACKNOWLEDGMENTS}

This work was supported by National Science Foundation of China (No. 81370979 and No. 81573478, to X. Zhang) and Shanghai Science and Technology Innovation (No. 1414090 3202 to X. Zhang). 


\section{REFERENCES}

Bentzen, B.H., Olesen, S.P., Ronn, L.C., and Grunnet, M. (2014). BK channel activators and their therapeutic perspectives. Front Physiol. 5, 389.

Black, J.A., and Waxman, S.G. (2013). Noncanonical roles of voltage-gated sodium channels. Neuron $80,280-291$

Bruderer, M., Richards, R.G., Alini, M., and Stoddart, M.J. (2014). Role and regulation of RUNX2 in osteogenesis. Eur. Cells Mater. 28, 269-286.

Chen, J., Zhang, W., Lin, J., Wang, F., Wu, M., Chen, C., Zheng, Y., Peng, X., Li, J., and Yuan, Z. (2014). An efficient antiviral strategy for targeting hepatitis $B$ virus genome using transcription activator-like effector nucleases. Mol. Ther. 22, 303-311.

Chen, Q., Tao, J., Hei, H., Li, F., Wang, Y., Peng, W., and Zhang, X. (2015). Up-regulatory effects of curcumin on large conductance $\mathrm{Ca}^{2+}$-activated $\mathrm{K}^{+}$channels. PLoS One 10, e0144800.

Contreras, G.F., Castillo, K., Enrique, N., Carrasquel-Ursulaez, W., Castillo, J.P., Milesi, V., Neely, A., Alvarez, O., Ferreira, G., Gonzalez, C., et al. (2013). A BK (Slo1) channel journey from molecule to physiology. Channels 7, 442-458.

Fernandez-Marino, A.I., Cidad, P., Zafra, D., Nocito, L., Dominguez, J., Olivan-Viquera, A., Kohler, R., Lopez-Lopez, J.R., PerezGarcia, M.T., Valverde, M.A., et al. (2015). Tungstate-targeting of BKalphabeta1 channels tunes ERK phosphorylation and cell proliferation in human vascular smooth muscle. PLoS One 10, e0118148.

Henney, N.C., Li, B., Elford, C., Reviriego, P., Campbell, A.K., Wann, K.T., and Evans, B.A. (2009) A large-conductance (BK) potassium channel subtype affects both growth and mineralization of human osteoblasts. Am. J. Physiol. Cell Physiol. 297, C1397-1408.

Hirukawa, K., Muraki, K., Ohya, S., Imaizumi, Y., and Togari, A. (2008). Electrophysiological properties of a novel $\mathrm{Ca}(2+)$ activated $\mathrm{K}(+)$ channel expressed in human osteoblasts. Calcif. Tissue Int. 83, 222-229.

Huang, H., Rao, Y., Sun, P., and Gong, L.W. (2002). Involvement of actin cytoskeleton in modulation of Ca21-activated $\mathrm{K}^{+}$channels from rat hippocampal CA1 pyramidal neurons. Neurosci. Lett. 332, 141-145.

Issa, N.P., and Hudspeth, A.J. (1994) Clustering of $\mathrm{Ca}^{2+}$ channels and $\mathrm{Ca}^{2+}$-activated $\mathrm{K}^{+}$channels at fluorescently labeled presynaptic active zones of hair cells. Proc. Natl. Acad. Sci. USA 91, 7578-7582

Jin, W., Sugaya, A., Tsuda, T., Ohguchi, H., and Sugaya, E. (2000). Relationship between large conductance calcium-activated potassium channel and bursting activity. Brain Res. 860, 21-28.

Kim, E.Y., Suh, J.M., Chiu, Y.H., and Dryer, SE. (2010). Regulation of podocyte BKCa channels by synaptopodin, Rho, and actin microfilament. Am. J. Physiol. 299, F594-F604.

Long, F. (2012). Building strong bones: molecular regulation of the osteoblast lineage. Nat. Rev. Mol. Cell Biol. 13, 27-38.

Lu, P., Chen, J., He, L., Ren, J., Chen, H., Rao, L., Zhuang, Q., Li, H., Li, L., Bao, L., et al. (2013). Generating hypoimmunogenic human embryonic stem cells by the disruption of beta 2microglobulin. Stem Cell Rev. 9, 806-813.
Mehranfard, N., Gholamipour-Badie, H., Motamedi, F., Janahmadi, M., and Naderi, N. (2015). Long-term increases in BK potassium channel underlie increased action potential firing in dentate granule neurons following pilocarpine-induced status epilepticus in rats. Neurosci. Lett. 585, 88-91.

Morera, F.J., Saravia, J., Pontigo, J.P., Vargas-Chacoff, L., Contreras, G.F., Pupo, A., Lorenzo, Y., Castillo, K., Tilegenova, C., Cuello, L.G., et al. (2015). Voltage-dependent BK and Hv1 channels expressed in non-excitable tissues: New therapeutics opportunities as targets in human diseases. Pharmacol Res. 101, 56-64.

Petkov, G.V. (2014). Central role of the BK channel in urinary bladder smooth muscle physiology and pathophysiology. Am. J. Physiol. R307, R571-584.

Sandow, S.L., and Grayson, T.H. (2009). Limits of isolation and culture: intact vascular endothelium and BKCa. Am. J. Physiol. Heart Circ. Physiol. 297, H1-H7.

Sausbier, U., Dullin, C., Missbach-Guentner, J., Kabagema, C., Flockerzie, K., Kuscher, G.M., Stuehmer, W., Neuhuber, W., Ruth, P., Alves, F., et al. (2011). Osteopenia due to enhanced cathepsin $\mathrm{K}$ release by BK channel ablation in osteoclasts. PLoS One 6, e21168.

Shen, R., Wang, X., Drissi, H., Liu, F., O'Keefe, R.J., and Chen, D. (2006). Cyclin D1-cdk4 induce runx2 ubiquitination and degradation. J. Biol. Chem. 281, 16347-16353.

Tokuda, S., and Furuse, M. (2015). Claudin-2 knockout by TALENmediated gene targeting in MDCK cells: claudin-2 independently determines the leaky property of tight junctions in MDCK cells. PLoS One 10, e0119869.

Wu, R.S., and Marx, S.O. (2010). The BK potassium channel in the vascular smooth muscle and kidney: alpha- and beta-subunits. Kidney Int. 78, 963-974.

Wu, Z., Yin, H., Liu, T., Yan, W., Li, Z., Chen, J., Chen, H., Wang, T., Jiang, Z., Zhou, W., et al. (2014) MiR-126-5p regulates osteoclast differentiation and bone resorption in giant cell tumor through inhibition of MMP-13. Biochem. Biophys. Res. Commun. 443, 944-949.

Yamamura, H., Ikeda, C., Suzuki, Y., Ohya, S., and Imaizumi, Y. (2012). Molecular assembly and dynamics of fluorescent proteintagged single KCa1.1 channel in expression system and vascular smooth muscle cells. Am. J. Physiol. Cell Physiol. 302, C1257C1268.

Yen, T.Y., Li, K.P., Ou, S.C., Shien, J.H., Lu, H.M., and Chang, P.C. (2015). Construction of an infectious plasmid clone of Muscovy duck parvovirus by TA cloning and creation of a partially attenuated strain. Avian Pathol. 44, 124-128.

Yu, S., Yerges-Armstrong, L.M., Chu, Y., Zmuda, J.M., and Zhang, Y. (2013). E2F1 effects on osteoblast differentiation and mineralization are mediated through up-regulation of frizzled-1. Bone 56 234-241.

Zhang, Y.Y., Yue, J., Che, H., Sun, H.Y., Tse, H.F., and Li, G.R. (2014). BKCa and hEag1 channels regulate cell proliferation and differentiation in human bone marrow-derived mesenchymal stem cells. J. Cell. Physiol. 229, 202-212. 